\title{
Stopping dynamics of a steady uniform granular flow over a rough incline
}

\author{
Stéphanie Deboeuf ${ }^{1 \star \star}$, Guillaume Saingier ${ }^{1,2}$, Nitharshini Thiruvalluvar ${ }^{1}$, Pierre-Yves Lagrée ${ }^{1}$, Stéphane Popinet ${ }^{1}$, \\ and Lydie Staron ${ }^{1}$ \\ ${ }^{1}$ Sorbonne Universités, UPMC Université Paris 6, CNRS UMR 7190, Institut Jean le Rond d'Alembert, F-75005 Paris, France \\ ${ }^{2}$ Surface du Verre et Interface, UMR 125, CNRS/Saint-Gobain, 93303 Aubervilliers, France
}

\begin{abstract}
Granular material flowing on complex topographies are ubiquitous in industrial and geophysical situations. Even model granular flows are difficult to understand and predict. Recently, the frictional rheology $\mu(I)$-describing the ratio of the shear stress to the normal stress as a function of the inertial number $I$, that compares inertial and confinement effects- allows unifying different configurations of granular flows. However it does not succeed in describing some phenomenologies, such as creep flow, deposit height, ... Is it attributable to the rheology, to non-local effects, ...? Here, we consider a thin layer of grains flowing steadily and uniformly on a rough incline, when the input mass flow rate is suddenly stopped. We focus on the arrest dynamics by using both experimental and numerical approaches. We measure the height and surface velocities of the granular layer during the long-time stopping dynamics and we compare our experimental results with computations of depthaveraged equations for a fluid of rheology $\mu(I)$.
\end{abstract}

\section{Introduction}

In order to be able to predict flows of divided matter encountered in the nature or in the industry, a first step is to gain in the understanding of small-scale controlled flows of model grains [1]. We study here a model gravitational flow of grains over an inclined plane, that is quasi one dimensional at first order [2]. We compare experimental results obtained with macroscopic hard frictional spheres and numerical results of depth-averaged equations in 1D of a thin continuous flow of frictional rheology $\mu(I)[2-$ 4]. This relates the friction coefficient $\mu$, equal to the ratio of the shear stress $\tau$ and the normal stress $P$, to the inertial number $I$, depending on the shear rate $\dot{\gamma}$ and the pressure $P$ :

$$
\mu(I)=\frac{\tau}{P}, \text { with } I=\frac{\dot{\gamma} d}{\sqrt{P / \rho}},
$$

$d$ and $\rho$ being the diameter and the density of grains. This friction coefficient allows to describe the dissipation occurring in the granular flow. Another way to write it, introducing a non Newtonian viscosity $\eta$, is [5]: $\tau=$ $\eta \dot{\gamma}$, with $\eta=\mu(I) P / \dot{\gamma}$. Predictions from the rheology $\mu(I)$ were already compared to some steady flows and some unsteady flows, such as spreading of a small volume of grains over an incline [6,7], initiation of granular surface flows in a narrow channel [8], or granular column collapses [5, 911]. The arrest of flowing grains was also studied in the case of a dam break [11] or flow over a slope [12, 13]. Here we focus on the stopping dynamics of a steady uniform flow over an incline when the mass flow rate vanishes suddenly.

\footnotetext{
^e-mail: sdeboeuf@dalembert.upmc.fr
}

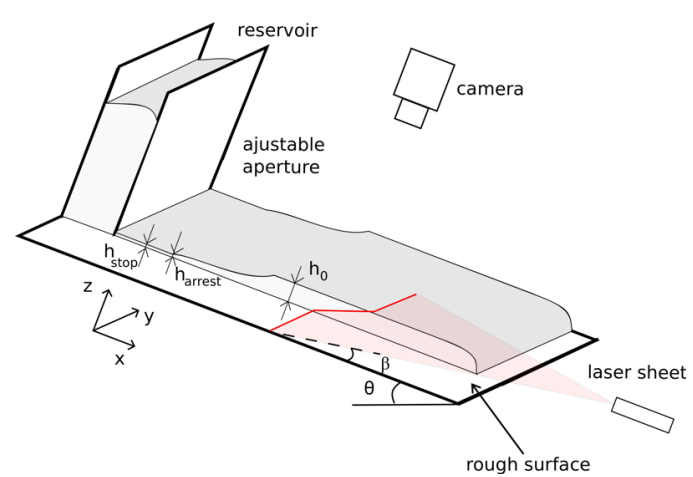

Figure 1. The reservoir aperture of an inclined plane can be suddenly closed to let the steady granular flow relax towards a static deposit

\section{Experiments}

The slope of a plane (Figure 1), $2 \mathrm{~m}$ long and $40 \mathrm{~cm}$ wide, with polyethylene plates for side walls, can be chosen at a fixed angle $\theta$. The aperture of a reservoir of grains at the top of the plane can be opened or closed quickly, allowing for the release or the arrest of a steady uniform granular flow. A gate allows to adjust the aperture thickness $h_{\text {gate }}$ in order to control the mass flow rate. The bottom surface of the plane is made rough by covering it with sand-paper of roughness about $200 \mu \mathrm{m}$. This boundary condition should allow for a zero slip velocity at the bottom. Grains are spherical glass beads of diameter $d=200 \pm 50 \mu \mathrm{m}$. This set-up allows for steady flows propagating at a constant velocity for some range of slope angles $\left(25^{\circ} \leq \theta \leq 35^{\circ}\right)$ 
and aperture thicknesses $\left(5 \mathrm{~mm} \leq h_{\text {gate }} \leq 30 \mathrm{~mm}\right)$. When opening the reservoir aperture, a granular front flows down the slope at constant velocity $u_{0}$ with a steady shape $h(x-$ $\left.u_{0} t\right)[14,15]$. Behind the front, the layer has a uniform height $h_{0}$. How this steady uniform flow stops when the aperture is suddenly closed?

Height profiles are measured thanks to laser profilometry: one laser sheet illuminates transversally the surface at a low incident angle, giving the temporal evolution of height $h(t)$ at a given position. About $1 \%$ of grains are dyed, allowing for the measurement of the surface velocity $\mathbf{u}_{s}(t)$ using a particle imaging velocimetry algorithm. To this aim, movies of size $1280 \times 720$ pixels with a spatial resolution of about 4 pixels $/ \mathrm{mm}$ at a frequency of $30 \mathrm{~Hz}$ are recorded. It is possible to infer the mean behaviour of the granular flow from surface kinematics $h$ and $\mathbf{u}_{s}(t)$, thanks to the zero slip velocity at the bottom, and assuming that the whole layer flows and is sheared. However this might be not true during the stopping dynamics.

\section{Numerical simulations}

In order to get predictions on the stopping of a flow for a frictional rheology $\mu(I)$, we solve numerically $1 \mathrm{D}$ depthaveraged equations [6]:

$$
\begin{gathered}
\frac{\partial h}{\partial t}+\frac{\partial}{\partial x}(h \bar{u})=0 \\
\frac{\partial}{\partial t}(h \bar{u})+\alpha \frac{\partial}{\partial x}\left(h \bar{u}^{2}\right)=h g \cos \theta\left(\tan \theta-\mu(\bar{I})-\frac{\partial h}{\partial x}\right),
\end{gathered}
$$

using the open source Navier-Stokes code initiated by S. Popinet (Basilisk) [16]. The streamwise and vertical coordinates are $x$ and $z, h$ is the thickness of the layer, $\bar{u}$ denotes the depth-averaged streamwise velocity and $g$ is the gravity acceleration. The granular layer is assumed to be thin enough, transversally uniform, and at a constant solid fraction $\phi \approx 0.60$ (incompressible). The shape factor $\alpha=\int_{0}^{h} u^{2}(z) d z /\left(h \bar{u}^{2}\right)$ is taken equal to $\alpha=1$, despite recent results that demonstrate the relevance to make other assumptions [15]. The right hand side terms of Eq. (3) are the gravity along the slope, the friction and the pressure gradient. The friction term can be interpreted either as a basal friction [6] or as the averaged internal friction over the thickness [17]. The friction $\mu(I)$ [4] is expressed as:

$$
\mu(I)=\mu_{0}+\frac{\Delta \mu}{I_{0} / I+1}
$$

where $\mu_{0}=0.41, \Delta \mu=0.35$ and $I_{0}=0.38$ were measured from experiments of uniform and steady flows and successfully compared with data of non uniform flow fronts for the same set-up [15]. The inertial number $\bar{I}$ in the depth-averaged approach is computed for $\alpha=1$ as:

$$
\bar{I}=\frac{\bar{u} d}{h \sqrt{\phi g h \cos \theta}} .
$$

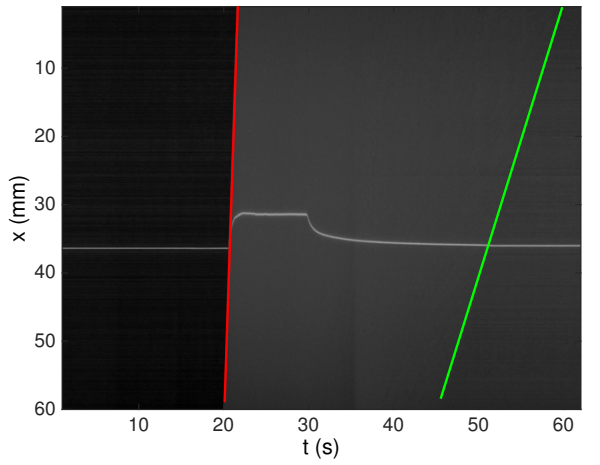

Figure 2. Space-time diagram showing the time evolution of one line of pixels taken along the streamwise direction $x$. The red and green lines indicate the velocity of the front and of the arrest wave respectively
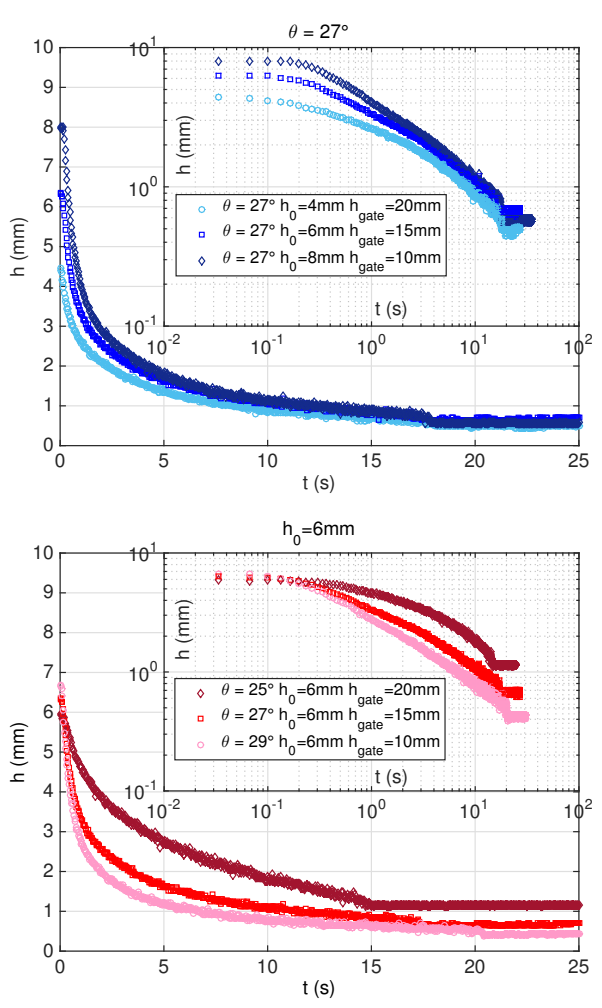

Figure 3. Time evolution of height $h(t)$ during the arrest of a flow when closing the reservoir gate from experiments at a constant slope angle $\theta=27^{\circ}$ for different thicknesses $h_{0}=4 \mathrm{~mm}$, $6 \mathrm{~mm}$ and $8 \mathrm{~mm}$ (a) and at a constant initial height $h_{0}=6 \mathrm{~mm}$ at different slope angles $\theta=25^{\circ}, 27^{\circ}, 29^{\circ}$ (b) in linear and logarithmic scales

\section{Transient stopping dynamics}

\subsection{Typical phenomenology}

The typical phenomenology of the transient stopping dynamics of a steady uniform granular flow is depicted in Figure 1 and shown in Figures 2 and 6 for experiments and simulations respectively. First a steady uniform flow over the incline is generated. Second the aperture gate is 


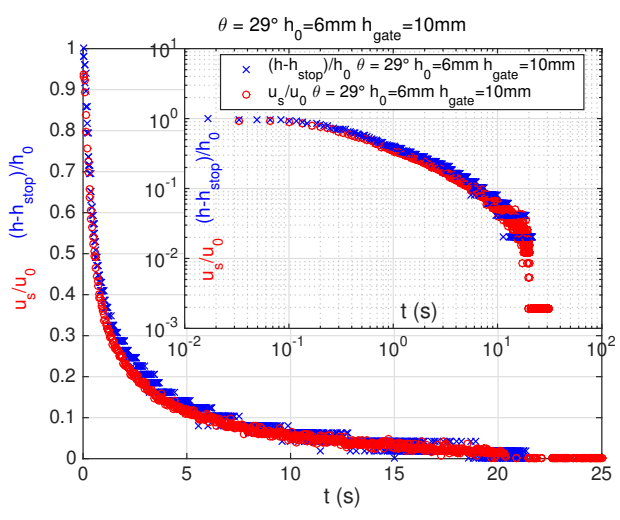

Figure 4. Time evolution of normalized height $\left(h(t)-h_{\text {stop }}\right) / h_{0}$ and normalized surface velocity $u_{s}(t) / u_{0}$ for one experiment at $\theta=29^{\circ}$ and $h_{0}=6 \mathrm{~mm}$ in linear and logarithmic scales

suddenly closed, inducing a boundary condition $h=0$ at the gate. This sudden variation of boundary condition creates a shock wave travelling at a velocity $u_{\text {shock }}$ larger than the front velocity $u_{0}$, then the height and the velocity decrease slowly. Finally, a deposit lays on the rough plane of uniform height $h_{\text {stop }}[18,19]$. It is known that $h_{\text {stop }}(\theta)$ is a function of the slope angle $\theta$ and depends on the properties of grains and of the bottom surface of the plane.

Another difference lays between experiments and simulations in the discontinuity of height observed experimentally at the end of the stopping dynamics: just before stopping, the still flowing height is equal to $h_{\text {arrest }}>h_{\text {stop }}$, that may be the signature of compressibility of the granular flow and its compression when stopping. By contrast, the height in the incompressible simulations continuously vanishes.

The stopping of the flow can be seen as the propagation of an arrest wave, defined as the transition between $u \neq 0$ and $u=0$. Figure 2 shows a space-time diagram: the shift of the projected laser sheet is proportional to the thickness $h(t)$. The granular flow front crosses the image at about $t \simeq 20 \mathrm{~s}$ with a velocity $u_{0}$ shown by the red line. The height is uniform after a few seconds. At about $t \simeq 30 \mathrm{~s}$, the shock wave generated by the closure of the gate crosses the image. Then the height decreases slowly for about 20s, when the arrest wave propagates at a velocity $u_{\text {arrest }}$, shown by the green line, larger than the front velocity $u_{0}$.

\subsection{Relaxation dynamics}

The height and the surface velocity decrease slowly after the passage of the shock wave (Figures 3 and ??). Figure 3 shows the height evolution during the stopping for different experiments realized at the same slope angle $\theta=27^{\circ}$ but different thicknesses $h_{0}=4 \mathrm{~mm}, 6 \mathrm{~mm}$ and $8 \mathrm{~mm}$ (a) and for the same uniform height $h_{0}=6 \mathrm{~mm}$ but at different slope angles $\theta=25^{\circ}, 27^{\circ}, 29^{\circ}$ (b). The typical time for the whole stopping of the granular layer is between $10 \mathrm{~s}$ and 100s. It seems that this time depends on the slope angle $\theta$ only, but not on the height $h_{0}$ or veloc-
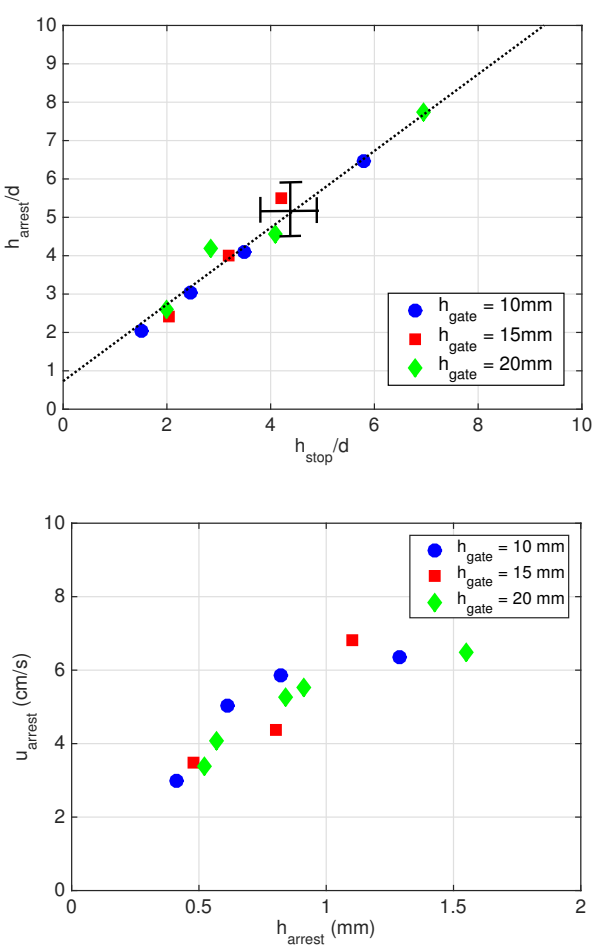

Figure 5. Experimental measurements of the arrest wave: height of the wave $h_{\text {arrest }}$ as a function of the deposit height $h_{\text {stop }}$ and velocity of the wave $u_{\text {arrest }}$ as a function of $h_{\text {arrest }}$

ity $u_{0}$ of the steady uniform flow. For now, we can not infer any obvious temporal evolution for $h(t)$ (exponential or power-law or logarithmic), but deeper studies are needed. While the height decreases until $h_{\text {stop }}$-the finite thickness of the deposit-, the surface velocity $u_{s}(t)$ continuously vanishes (Figure 4). The temporal evolution of $h(t)$ and $u_{s}(t)$ are exactly the same, as shown by Figure 4, where normalized surface velocity $u_{s}(t) / u_{0}$ and normalized height $\left(h(t)-h_{\text {stop }}\right) / h_{0}$ have been plotted for one experiment.

We measured systematically the experimental values of the height $h_{\text {arrest }}$ and the velocity $u_{\text {arrest }}$ of the arrest wave, shown in Figure 5: it seems that they depend only on the slope angle $\theta$. Just before stopping, the still flowing height $h_{\text {arrest }}$ is not equal to the deposit thickness $h_{\text {stop }}$. Figure 5a shows that $h_{\text {arrest }}=h_{\text {stop }}+\delta h$ with $\delta h \simeq 1 d$. The fact that the compression $\delta h$ does not change with the thickness of the flow but is constant, may indicate that the arrest of the flow may not occur as a whole but may happen through the decrease of the thickness of flowing grains. Also, $h_{\text {arrest }}$ and $u_{\text {arrest }}$ are correlated.

One advantage of numerical simulations is to get the whole spatial and temporal evolution of the granular flow thickness. Figure 6 shows the spatial profile of the granular layer at different times $t$ in the incline framework $h(x)$ (a), in the front framework $h\left(x-u_{0} t\right)$ (b) and in the shock wave framework $h\left(x-u_{\text {shock }} t\right)$ (c). For the present simulation, the shock wave propagates at a velocity $u_{\text {shock }}=$ $2.3 u_{0}$, approximately twice larger than the front velocity 

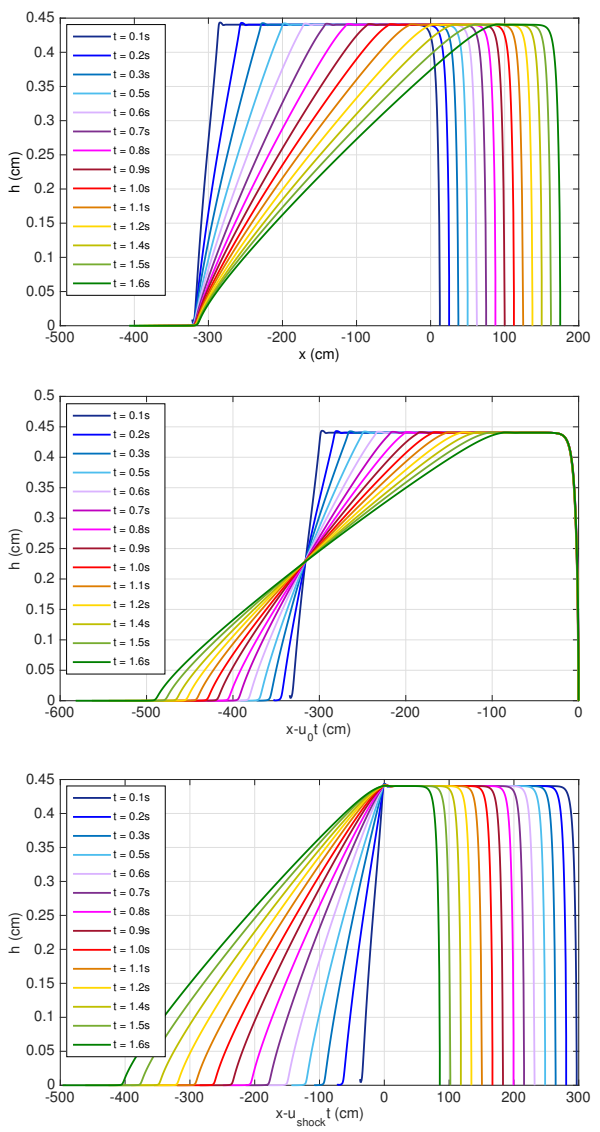

Figure 6. Streamwise height profiles from one numerical simulation at different times: $h(x)$ in the incline framework, $h\left(x-u_{0} t\right)$ in the front framework and $h\left(x-u_{\text {shock }} t\right)$ in the shock wave framework.

$u_{0}$. The arrest wave and the shock wave have the same velocity: $u_{\text {shock }}=u_{\text {arrest }}$ but propagate in opposite directions. As for a Newtonian fluid and for a fluid at constant friction $\mu$ in the case of a dam-break [11], all profiles in the front framework $h\left(x-u_{0} t\right)$ cross at the same point at first order (Figure 6b). Figure 6c suggests to use the same tools as for describing the spreading of a dam-break by taking into account the velocity propagation of the shock wave.

\section{Conclusion}

We study the long-time stopping dynamics of a steady uniform granular flow over a rough incline plane by using experimental and numerical tools. The stopping dynamics exhibit the propagation of a shock wave and of an arrest wave. More investigations are still needed to know more about the internal dynamics of the flow during the stopping. A possibility would be to measure velocity profiles through transparent lateral walls, to design a two dimensional flow or to use discrete element methods.

As the local frictional rheology $\mu(I)$ does not predict a static deposit or a height threshold, that is one major lim- itation of $\mu(I)$ until today, alternatives would be to consider a non-local rheology $[20,21]$, or to write the friction law as a function of $I$ and $h: \mu(I, h)[7,22]$. We made some experiments with a smooth bottom plane. In this case, there is almost no deposit $\left(h_{\text {stop }} \simeq 0\right)$, whatever the slope angle is. However, the flowing and stopping phenomenology are the same as for a rough bottom surface, suggesting that there is actually shear inside the granular layer in the steady uniform regime even in the case of a smooth bottom, that would allow for closer comparisons with predictions of $\mu(I)$ in the stopping regime.

\section{References}

[1] B. Andreotti, Y. Forterre, and O. Pouliquen, Granular Media: Between Fluid and Solid (Cambridge University Press, Cambridge, 2013)

[2] GDR MiDi, Eur. Phys. J. E, 14 341-365 (2004)

[3] F. da Cruz, S. Emam, M. Prochnow, J.-N. Roux, and F. Chevoir, Phys. Rev. E 72021309 (2005)

[4] P. Jop, Y. Forterre, and O. Pouliquen, Nature 44 727-730 (2006)

[5] P.-Y. Lagrée, L. Staron, and S. Popinet, J. Fluid Mech., 686 378-408 (2011)

[6] S. B. Savage and K. Hutter, J. Fluid Mech., 199 177-215 (1989)

[7] O. Pouliquen and Y. Forterre, J. Fluid Mech., 453 133-151 (2002)

[8] P. Jop, Y. Forterre, O. Pouliquen, Phys. Fluids 19 088102 (2007)

[9] E. Lajeunesse, A. Mangeney-Castelnau, and J. P. Vilotte, Phys. Fluids, 162371 (2004)

[10] G. Lube, H.E. Huppert, R.S.J. Sparks, and M.A. Hallworth, J. Fluid Mech., 508 175-199 (2004)

[11] R. R. Kerswell, Phys. Fluids, 17057101 (2005)

[12] S. Douady, B. Andreotti, P. Cladé, and A. Daerr, Adv. Compl. Syst. 4 509-522 (2001)

[13] T. Boutreux and P.-G. de Gennes, C. R. Acad. Sci. Paris, Série II b 326 257-262 (1998)

[14] O. Pouliquen, Phys. Fluids 111956 (1999)

[15] G. Saingier, S. Deboeuf, and P.-Y. Lagrée, Phys. Fluids, 28053302 (2016)

[16] S. Popinet, J. Comput. Phys., 190 572-600 (2003)

[17] J. M. N. T. Gray, and A. N. Edwards, J. Fluid Mech. 755 503-734 (2014)

[18] O. Pouliquen, and N. Renaut, Journal de Physique II, EDP Sciences, 6 923-935 (1996)

[19] A. Daerr and S. Douady, Nature, 399 241-243 (199)

[20] M. Bouzid, M. Trulsson, P. Claudin, E. Clément, and B. Andreottti, Phys. Rev. Lett., 111238301 (2013)

[21] K. Kamrin and D.L. Henann, Soft Matter, 11179 (2015)

[22] A. Mangeney, F. Bouchut, N. Thomas, J.-P. Vilotte, and M.-O. Bristeau, J. Geophys. Res. 11202017 (2007) 